\title{
Das klimaethische Selbst als Subjektivierungsform
}

\section{Eine idealtypische Konstruktion}

\author{
Alexandria Krug \\ Journal für Psychologie, 28(2), 171-190 \\ https://doi.org/10.30820/0942-2285-2020-2-171 \\ CC BY-NC-ND 3.0 DE \\ www.journal-fuer-psychologie.de
}

\begin{abstract}
Zusammenfassung
In diesem Beitrag wird in einem theoretisch-normativen Diskurs aus der Perspektive der angewandten Ethik den Moralisierungstendenzen im Kontext der Klimawandelproblematik nachgegangen. Ziel ist es, sich den Ausdrucksformen der Moral und des Moralisierens in klimaethischer und verantwortungstheoretischer Sicht zu nähern. Die im Alltagsdiskurs zunehmende, normativ fordernde Moralisierung hin zu einem klimaverantwortlich handelnden Individuum ist an Verantwortungsdiskurse und Argumentationen der Klimaethik gebunden. Diese gilt es zu explizieren und zu prüfen, um die Ansprüche der Klimamoral als Selbstthematisierung zu ergründen. Fokussierend wird auf die in diesem Beitrag idealtypisch konstruierte Subjektivierungsform des klimaethischen Selbst hingearbeitet, um eine theoretische Fundierung dieser spezifisch normativen Subjektivierungsform als Diskussionsgegenstand und Ansatzpunkt für weitere moralpsychologische Forschung und transdisziplinäre Diskurse zu skizzieren.
\end{abstract}

Schlüsselwörter: Klimaethik, Verantwortung, klimaethisches Selbst, moralisierende Subjektivierungsform, idealtypische Konstruktion

\section{Summary}

The climatic-ethical self as a form of subjectivation. An ideal-typical construction In this article, the moralization tendencies in the context of the climate change problem are investigated in a theoretical-normative discourse from the perspective of applied ethics. The aim is to approach the expressions of morality and moralization from a climate ethical and responsibility theoretical perspective. The increasing, normatively demanding moralization in everyday discourse towards an individual acting responsibly for the climate is bound to the discourses and arguments of climate ethics. These must be explained and examined in order to fathom the claims of »climate morality « as a self-topic. This article focuses on the ideal 
type of subjectivation of the climatic ethical self, in order to outline a theoretical foundation of this specifically normative form of subjectivation as a subject of discussion and starting point for further moral psychological research and transdisciplinary discourses.

Keywords: Climate ethics, responsibility, climatic ethical self, moralizing form of subjectification, ideal typical construction

\section{Der Balanceakt der Moral - Moralisierungstendenzen im Klimawandeldiskurs}

Täglich vollziehen wir Handlungen und treffen Entscheidungen, die - zumindest prinzipiell - auch hinsichtlich des Klimawandeldiskurses und des Anspruchs eines möglichst klimaverantwortlichen Verhaltens des Individuums betrachtet werden können. Dabei werden im privaten und öffentlichen Leben von politischer, bildungsbezogener, gesellschaftlicher, medialer, wissenschaftlicher und wirtschaftlicher Betrachtungsebene zahlreiche implizite und explizite Anforderungen an das Individuum gestellt. Diese Anforderungen an das Subjekt sind in der Klimawandelproblematik nicht selten mit moralischen Ansprüchen und normativ-ethischen Verfärbungen verknüpft. Besonders in der Klimawandeldebatte erfolgt eine Moralisierung, in » welcher das Individuum zusehends für hoch abstrakte und allgemeine Anliegen moralisch verantwortlich gemacht wird und sich auch verantwortlich fühlt « (Knobloch 2015, 182). Der Klimawandeldiskurs ist $\gg$ filled with moral claims « (Besio und Pronzini 2014, 287). Sei es in der Bildungslandschaft, speziell die »Bildung für nachhaltige Entwicklung « (vgl. BMBF 2019; Bendig 2018), oder der stete Diskurs um Ernährungs- und Mobilitätsweisen, Konsumverhalten und Energieverbrauch (vgl. U. Schmidt 2019). Knobloch $(2015,170)$ formuliert es zugespitzt: »Naturschutz, Klimaschutz, Fairness im Handel sind solche fraglosen Hochwerte, die jedes mit ihnen verknüpfte Produkt moralisch adeln. «In vielen Domänen des alltäglichen Lebens der westlichen Industrienationen und insbesondere innerhalb wohlhabender, bildungsbürgerlicher Schichten werden klimaverträgliche Normen und Praxen verhandelt, die mehr oder weniger stark den Anspruch einer moralischen Pflicht oder zumindest einer moralischen Handlungsempfehlung erheben und zu Bezugspunkten der subjektivierenden Selbstthematisierung avancieren (vgl. Neuhäuser 2020; Compagna 2020). Der Klimawandel wird insbesondere medial als Risiko konstruiert, das in moralische Ansprüche transferiert wird (vgl. A. Schmidt 2015, 200-10). »[W]issenschaftliches Wissen und Sachargumente [werden] mit Dramatisierungen von Problemdringlichkeiten und Moralisierungen des Handlungsbedarfs verknüpft « (Keller 2008, 286). Moral und Ethik werden zu Diskursformen, die in der Klimawandeldebatte eine kommunikative und subjektivierende Rolle einnehmen und Polarisierungen bedingen. 
»Moral und Ethik werden entweder verehrt oder sehr skeptisch betrachtet. Während einige Autoren argumentieren, dass sich die Lösung des Klimawandelproblems im kollektiven moralischen Verhalten von Ländern, Industrien und allen Bürgern befindet [...], betonen andere, dass Politiker und Unternehmen Moral aus einem ausschließlich opportunistischen Blickwinkel betrachten und sie instrumentalisieren, um ihr Image zu polieren « (Besio und Pronzini 2018, 59f.).

Moralische Ansprüche werden damit zu divergent genutzten Instrumenten innerhalb der Klimawandelproblematik. Nicht nur Unternehmen, Institutionen und globale Akteure*innen stehen im Fokus, sondern auch die Individuen werden in ihrer Eigenverantwortung angesprochen. Moralische Anforderungen im Sinne eines klimaverträglichen und nachhaltigen Lebensstils und Daseins fungieren zunehmend als Vehikel der Subjektvierung. »Die Zuschreibung von Mitverantwortung und damit implizit auch Mitschuld an der drohenden klimabedingten Katastrophe erfolgt unmissverständlich als Appell, der bereits als Subjektivierung der Nachhaltigkeit von Pritz (2018) plausibel beschrieben worden ist « (Compagna 2020, 41). Mit dieser Individualisierung des Geltungs- und Anforderungsbereichs klimaverträglicher, klimaverantwortlicher und damit mitweltlicher Handlungs- und Denkdevisen wird eine transformierende Moralisierung des Individuums vollzogen (vgl. Compagna 2020). »Das Klima markiert die neue Gretchenfrage der Moral, die Produktion von $\mathrm{CO}_{2}$ wurde zum neuen, leicht quantifizierbaren Maßstab für Gut und Böse, der ökologische Fußabdruck definiert den moralischen Status eines Menschen heute « (Liessmann 2019). Gleichzeitig zeigen Studien, dass sich große Teile der deutschen Bevölkerung ein persönliches Verantwortungsgefühl hinsichtlich des Klimawandels zuschreiben und dieser mit Blick auf universale Gerechtigkeit, globale Ungleichheit und ökonomische Problematiken betrachtet wird (vgl. Lörcher 2019, 56f.). In dieser Darstellung deutet sich die Problematik der moralischen Verortung des Menschen im Klimawandeldiskurs an: der Balanceakt zwischen Moral und Moralismus. »Wenn Debatten moralisch werden, verhärten sich schnell die Fronten. Dann steht Haltung gegen Haltung, denn geht es um > Gut< oder $>$ Böse <, nicht um die produktive Auseinandersetzung, um die Lösung eines Problems « (Vašek 2019, 16). Die Gleichsetzung des moralischen Status eines Menschen mit seinem klimabezogenen Verhalten ist eine reduktionistische Sichtweise, die zu einer polarisierten Dualität von Moral und Moralismus führen kann (vgl. Neuhäuser 2020; BoehmeNeßler 2019). Eine Abwendung von der Moral und einem moralisch-ethischen Diskurs auf einer lebenspraktischen Ebene im Spannungsfeld der Klimawandelproblematik und der Verortung des Menschen in ihr erscheint indes als keine überzeugende Option, denn Moral gehört als Diskurspunkt zum menschlichen Leben (vgl. Bischof 2012). Deswegen gilt es zu explizieren, welche Gründe für eine moralische Betrachtungsweise hinsichtlich des Klimawandels und des auf ihn bezogenen menschlichen Handelns 
vorliegen und wie diese das menschliche Selbst kennzeichnen können beziehungsweise welche Synergien in einer klimaethischen Perspektive zwischen Moral, Verantwortung und dem handelnden Individuum hinsichtlich des Klimawandeldiskurses bestehen.

\subsection{Konzeptionelles Vorgehen}

Der vorliegende Beitrag versucht sich dem in drei Schritten zu nähern. Zunächst werden exemplarische Ansätze der Klimaethik vorgestellt, um diese in Verbindung mit moralischen Überlegungen zu bringen. Zudem werden hier zentrale Perspektiven des Diskurses um die Verantwortung skizziert. Diese theoretisch-normative Fundierung dient als Basis für die Konstruktion der idealtypischen Subjektivierungsform des klimaethischen Selbst, welche anschließend dargelegt wird. In einem abschließenden Betrachtungsschritt werden Fragestellungen aus dieser subjektivierenden Thematisierung des Selbst für die moralpsychologische Forschung und die Psychologie des Selbst entwickelt. Diese Konstruktion erfolgt als theoretischer, ethisch-normativer Entwurf. Die empirische Einbettung in biografische, narrative, soziokulturelle und affektive sowie ökonomische Domänen menschlichen Handelns sind nicht Bestandteil dieses Beitrags.

\subsection{Grundlegende Bestimmungen des Selbst}

Das hier idealtypisch zu konstruierende klimaethische Selbst soll als ein (selbst-)reflexives, am demokratischen und klimaorientierten Diskurs potenziell kritisch teilhabendes und partizipativ befähigtes Selbst verstanden werden (vgl. Schroer 2006, 44). Es wird zudem als adaptionsfähig und sich prozesshaft in Auseinandersetzung mit der Umwelt und sich selbst stetig modifizierend entworfen (vgl. Greve und Etzold 2018, 14f.). Ferner wird hier ein Begriff des Selbst verwendet, der von einem Selbst als wissendes und selbstbezügliches »Entwicklungssystem « (Greve und Etzold 2018, 14) ausgeht. Zudem wird in Anlehnung an Luhmann (1993) an die Idee unterschiedlicher und vielfältiger Selbstformen als Ausgestaltungsfacetten des Gesamtselbst angeschlossen, um das ideale klimaethische Selbst als mögliche Facette eines Subjekts greifbar zu machen, das als adaptive Selbstthematisierung verstanden werden kann (vgl. Schroer 2006, 54). Die Konstruktion und Gestaltung des Selbst ist dabei bestimmt von selbstthematisierenden Reflexionsund Erfahrungsintegrationsprozessen des sich selbst mit konstituierenden Subjekts bei gleichzeitiger Adaption sozialer Interaktionserfahrungen und diverser Ansprüche der Mitwelt (vgl. Kessler und Fritsche 2018, 71ff.). Dies steht in enger Vernetzung zur angedeuteten Selbstthematisierung und Subjektivierung, die durch moralisierende Tendenzen im Alltag und eigene Reflexionsfähigkeiten gefördert werden. 


\subsection{Grundlegende Bestimmungen der Selbstthematisierung und Subjektivierung}

Unter Selbstthematisierung wird hier der $\gg \operatorname{reflexiv}[\mathrm{e}]$ und kommunikativ[e] Bezug des Subjekts auf sich selbst « (Willems und Pranz 2008, 189) verstanden. Das Subjekt macht sich selbst zum Thema. Es ist in der Lage, sein eigenes Selbst zu hinterfragen und sich selbst bewusst zu machen (vgl. Burkart 2006, 18). Der Diskurs um machttheoretische und systemische Wirkungen der Selbstthematisierung und Subjektivierung, wie sie unter anderen von Foucault (1993) oder auch Althusser (2010) dargelegt worden sind, spielt in dieser Konstruktion allenfalls eine sekundäre Rolle. Willems und Pranz $(2008,191)$ greifen diesen Punkt in kritischer Weise auf und konstatieren, dass sich die modernen (moralischen und lebenspraktischen) Anforderungen an Individuen im semantischen Kreis von Freiheit, Individualität und Autonomie sowie Reflexivität bewegen. Zuschreibungen und Selbstthematisierungen fußen auf hochgradig selbstbezüglichen Entwicklungstendenzen und -anforderungen. Dieser Kritik soll hier aus einer anderen Perspektive bei gleichzeitiger Kenntnis von Optimierungsformen (vgl. Bröckling 2007), macht- und reproduktionsorientierten Ansätzen (vgl. Althusser 2010) und anderen Subjektivierungsformen (vgl. Bruder 2005) begegnet werden. »Die Frage [...] ist vor diesem Hintergrund, wie Akteur/innen auf unterschiedliche Ordnungen eines idealen Subjekt-Seins (auch jenseits ökonomischer Appellstrukturen und Aktivierungsmuster) Bezug nehmen « (Geimer et al. 2019, 3). Es handelt sich so um eine idealtypische Subjektivierungsform, die indes jedem Menschen qua Menschsein und Fähigkeit zum kritischen Denken zugänglich sein kann und explizit nicht als »weitere Verschärfung der Selbstdisziplinierung « (Compagna 2020, 41) verstanden werden soll.

\subsection{Grundlegendes Anliegen}

Die Idee ist vielmehr zu zeigen, dass es durchaus virulente »[n]ormativ aufgeladene, diskursiv-hegemoniale Apellstrukturen « (Geimer und Amling 2019, 20) in der Klimawandeldebatte gibt, diese aber mit einem Blick in klimaethische und verantwortungstheoretische Argumentationsgebäude zum Teil begründbar und damit überzeugend sein können. Die Thematisierung des Selbst als klimaethisches Selbst ist damit eine doppelte Konstruktionsleistung. Zum einen ist es die mehr oder weniger unhinterfragte Integration, Affirmation und Repräsentation externer, moralischer Fremdanforderungen an ein klimaverträgliches Subjekt durch dieses selbst als Selbstanforderungen und zum anderen ist es die Prüfung dieser normativen Ansprüche und deren idealtypische Konstruktion in Form einer idealen Facette der Selbstthematisierung, die durch den Selbstbezug des Subjekts seiner eigenen kritischen Prüfung unterliegen kann. Die 
Möglichkeit dieser idealtypischen Konstruktion liegt in der Transparentmachung und Vergegenwärtigung dieser Prozesse, die das klimaethische Selbst konstituieren. Diese idealtypische Konstruktion ist aus normativen und ursprünglich bildungstheoretischpädagogischen Überlegungen bezüglich der Verortung und potenziellen Handlungsfähigkeit des Individuums im Klimawandeldiskurs erwachsen. Sie ist geprägt von der normativen Auffassung der Autorin, dass eine kritisch-konstruktive und reflexiv-ethische Orientierung des Individuums zur gegenwarts- und zukunftsfähigen Verortung desselben im Klimawandeldiskurs beitragen kann. Darauf, dass der hier gewählte Zugang, der vornehmlich auf Rationalität, Reflexivität, Ein- und Umsicht baut und den Gegenstand darüber hinaus positiv konnotiert, selbstredend bisweilen in seiner Idealtypik vielleicht sogar idealisiert, sei an dieser Stelle zumindest hingewiesen (vgl. Compagna 2020).

\section{Moral, Klimaethik, Gerechtigkeit und Verantwortung - Die Verortung des Klimawandels im philosophischen Diskurs}

\subsection{Moral}

Das Individuum sieht sich im Klimawandeldiskurs nicht allzu selten in einer Lage der Überforderung, des Nicht-Verstehens, der Diffusion und Dualität von Verantwortung und Ignoranz in Form einer abwehrenden Relativierungshaltung. Zahlreiche Studien zeigen, dass viele Menschen ein Wissen vom Klimawandel besitzen und ihn als Umweltproblem wahrnehmen, aber ihn aufgrund seiner Komplexität als räumlich und zeitlich entferntes Problem einstufen (vgl. Pfister et al. 2017; Beyerl 2010; Böhm 2008). Sterman (2008) konnte aufzeigen, dass die komplexen Kausalketten innerhalb des Klimawandels von Erwachsenen nur marginal wahrgenommen und verstanden werden, was zu gewissen Fehlkonzepten führt, die eine abwartende Haltung begünstigen. Aktuellere Studien weisen aber darauf hin, dass sich unter anderen die deutsche Bevölkerung in stärkerem Maße eine Verantwortung gegenüber dem Klimawandel zuschreibt und ein klimafreundliches Verhalten als positive Handlung ansieht (vgl. Lörcher 2019, 58). Auch in internationaler Perspektive zeigt sich eine enge Kopplung von moralischen Ansichten und der Einschätzung des Klimawandels. Klimaschutz und klimafreundliches Agieren werden vermehrt als Fragen und Angelegenheiten der Moral deklariert (vgl. im Überblick A. Schmidt 2015, 80f.). Die moralisierenden Selbstthematisierungstendenzen sind damit oberflächlich erfasst, verweisen aber auf eine prinzipielle moralische Konnotation der Klimawandeldebatte (vgl. Compagna 2020). Neben den umweltpsychologischen, sozialpsychologischen und denen aus der Risikowahrnehmungsforschung stammenden Arbeiten wird sich an dieser Stelle dem philosophischen Problem des Kli- 
mawandels zugewandt, um die moralisierenden Tendenzen und Ansprüche an das Subjekt theoretisch und ethisch zu prüfen. Zunächst stellt sich dabei die Frage, ob der Klimawandel überhaupt ein moralisches Problem ist. Wenn der Klimawandel eine ethische Herausforderung ist oder zu einer wird, dann betrifft er uns als Menschen und damit unser Handeln in Bezug auf ihn (vgl. Roser und Seidel 2015). Die analytische Darlegung dieser Relation ist geprägt von der Synergie von Moral, Ethik und Gerechtigkeit sowie Verantwortung, die allesamt Diskursfolien der Moralisierungstendenzen des Individuums auf abstrakter Ebene sind. Die an das Individuum gerichteten SollensSätze sind normative Sätze. Der Fleischkonsum soll eingeschränkt werden, das Fliegen soll vermieden werden etc. Wir müssen das Klima schützen. All dies sind Aussagen, die einen Anspruch auf eine ultimative Wahrheit erheben. Sie klassifizieren Handlungen per se als gute oder schlechte Handlungen. Das klingt nach einer allzu simplen und damit angreifbaren Logik für die Skeptiker*innen einer moralischen Verantwortung gegenüber dem Klimawandel, die durch die Fokussierung auf den reinen Moralismus die graduellen und für den offenen Diskurs zwingend notwendigen Abstufungen in den Moralisierungstendenzen übersehen (vgl. Neuhäuser 2020). »Die Moral regelt unser zwischenmenschliches Verhalten, sie sagt uns, wie wir mit anderen umgehen sollen - zum Beispiel, dass Lügen nicht in Ordnung ist. Als >Moralisten $<$ im abwertenden Sinn bezeichnen wir hingegen jemand, der alles in übertriebener Weise > moralisierend $<$ beurteilt « (Vašek 2019, 17). Darin liegt bereits eine bedeutende graduelle Unterscheidung. Moralistische und dogmatische Moralisierungstendenzen erheben den Anspruch auf Absolutheit. Moral als solche ist aber vielmehr auch ein Leitfaden. Vašek $(2019,17)$ beschreibt dies treffend:

»Fast niemand zweifelt daran, dass wir wenigstens minimale moralische Maßstäbe brauchen, um das menschliche Zusammenleben zu regeln. Was den Moralisten ausmacht, das ist, dass er kaum andere Maßstäbe kennt. Der Moralist überdehnt also den Geltungsbereich der Moral, er teilt gleichsam alle in $>$ gut $<$ oder $>$ böse $<$ ein, damit tendiert er zu Selbstgerechtigkeit und Intoleranz.«

Was nützt das für die Betrachtung des Klimawandels und des Verhaltens des Menschen in Bezug auf ihn? Zunächst sensibilisiert es für eine begriffliche Klarheit und die notwendige Transparenz des potenziellen Wirkspektrums der Moralisierung. Außerdem verweist dies auf eine ethische Auseinandersetzung, die einen sachlicheren Blick auf die Annahmen der moralischen Perspektiven des Klimawandels liefern kann (vgl. Rehmann-Sutter 2019). Berger (2011) skizziert die Potenziale und Grenzen einer solchen Auseinandersetzung: »Eine Ethik im Klimawandel hilft nur in der Klärung von Normen und Maßstäben, wie sie unabhängig von spezifischen Zugehörigkeiten und Loyalitäten verallgemeinerbar sind. Das ist allein nicht wenig.« 


\subsection{Klimaethik}

Wie bereits erwähnt stellt sich die basale Frage nach der moralischen Einordnung des Klimawandels als erste Prämisse. Wieso stellt uns der Klimawandel vor eine vermeintliche ethische Herausforderung? Wenn wir moralische Fragen als »normative Fragen « verstehen, bei denen es darum geht zu klären, was man tun soll, » was gerecht ist, wozu wir verpflichtet sind, was erlaubt und was verboten ist « (Roser und Seidel 2015, 1), dann unterliegt der Klimawandel einem moralischen Betrachtungsfeld in allen Diskursbereichen. Der Klimawandel ist weiterhin ein faktisches Phänomen der natürlichen Welt. Eine Ist-und-Wird-Dynamik, die durch menschliches Handeln respektive die mit diesem einhergehenden Emissionen hervorgerufen wird und damit den Status eines reinen »Naturphänomens « in vielerlei Hinsicht verliert (vgl. Schönwiese 2019; Rahmstorf und Schellnhuber 2019).

»Beim Klimawandel liegt der Fall [nämlich] anders: Ein >natürliches< Phänomen ist der Klimawandel nur insofern, als er > in der Natur < stattfindet; anders als die Mondbewegung ist der Klimawandel zu einem substanziellen Teil menschengemacht und kann entsprechend durch menschliches Handeln gestoppt, verlangsamt oder beschleunigt werden « (Roser und Seidel 2015, 2).

Dies bedeutet, dass der Mensch und die Gesellschaft in mehr oder weniger direktem Wirkzusammenhang mit dem Klimawandel stehen (vgl. Schönwiese 2019, 77-88). Genau hier liegt ein Problem: Die direkten Auswirkungen und langfristigen Folgen des Klimawandels sind nicht unmittelbar, sondern es liegt eine »zeitliche Kluft zwischen Ursache und Wirkung « (Roser und Seidel 2015, 7) vor. Dies führt zu einer schwierigeren Bewertungssituation, denn unsere auf den Nahbereich fokussierte alltägliche Ethik kommt hier an ihre Grenzen. Ein weiteres Problem ist die räumliche Distanz der Ursache-Wirkungs-Zusammenhänge, denn vielfältige Auswirkungen des Klimawandels finden in anderen Regionen der Welt statt, zu denen viele Menschen keinen unmittelbaren Kontakt haben. Das bedeutet, dass der Klimawandel ein globales Problem der moralischen Betrachtung ist und diese räumliche und zeitliche Ausdehnung erschwert den direkten und kontinuierlicheren Argumentationsgang einer Alltagsethik. Wenn beispielsweise eine SUV-Fahrerin den Wagen nutzt, dann obliegen dieser Person die Vorteile, aber die Nachteile fallen Personen zu, die in anderen Gegenden der Welt leben oder noch gar nicht geboren sind. Der Klimawandel reist damit die direkte UrsacheWirkungs-Kette einer einsichtigen und alltagstauglichen Ethik auseinander, strapaziert und dehnt sie. Dies hängt damit zusammen, dass viele der jetzigen Handlungen erst in Jahrzehnten oder Jahrhunderten ihre Auswirkungen zeigen werden und die direkte Zurechenbarkeit der Relation von Handlungen und deren Folgen kaum gegeben ist und 
sein wird. Der Klimawandel wird zum intergenerationellen und gerechtigkeitsbasierten Problemfall.

\subsection{Klimagerechtigkeit}

Der Klimawandel ist als globales Phänomen ein Problem globaler Ungerechtigkeit, die durch seine zeitliche Dimension verstärkt wird (vgl. Roser und Seidel 2015, 6ff.). »Im Mittelpunkt der bisherigen Klimaethik steht die Klimagerechtigkeit « (Gesang 2011, 47). Betrachtet man dabei die drei prominentesten Ansätze der Klimagerechtigkeit, die hier exemplarisch erläutert werden sollen, dann fokussiert man sich auf die moralischen Grundlagen dieser und behandelt ausschließlich Fragen der generativen Gerechtigkeit. Formen der sozialen Gerechtigkeit spielen in den folgenden Prinzipien eine untergeordnete Rolle.

Als in öffentlichen Diskursen bekanntes Prinzip ist das Gleichheitsprinzip zu nennen. Dieses egalitaristische Prinzip sieht in einer relationalen Gleichheit alle Akteur*innen etwas intrinsisch Gutes. In einer kritischen Perspektive muss konstatiert werden, dass die Herstellung und Aufrechterhaltung eines gleichen Woblstands für alle zu jeder Zeit aber lebensfremd erscheinen. Insgesamt wird dieses Prinzip als zu wenig praktikabel eingestuft (vgl. Gesang 2011, 48f.). Neben dieser Position existiert das Schwellenwertprinzip, das sowohl mit Annahmen eines absoluten als auch eines relativen Schwellenwerts operiert. Die notwendige und hinreichende Bedingung für die Gewährleistung von Gerechtigkeit wäre erfüllt, wenn »gegenwärtige und zukünftige Generationen > genug < haben, also einen bestimmten Schwellenwert bzgl. des Wohlergehens überschreiten « (Gesang 2011, 52). Die grundlegende Kritik an diesem Prinzip besteht darin, dass das Maß und das Ansetzen eines Schwellenwerts ein innerlogisches Gerechtigkeitsproblem an sich sind, da aufgrund diverser Unterschiedlichkeiten und immanenter Veränderungen dieser nie global stabil gehalten werde könne (vgl. Gesang 2011, 53ff.). Als drittes Prinzip sei das Vorrangsprinzip (Prioritarismus) benannt. Gerechtigkeit umfasst hier den moralischen Grundsatz, dass die Armen in den Blick genommen werden und alles unternommen wird, um ihre Vulnerabilität in Bezug auf den Klimawandel zu senken. Die Industrieländer müssten aufgrund ihrer primären Verantwortung für den Klimawandel den betroffenen Ländern in der Entwicklung und Anpassung an den Klimawandel helfen. Grundsätzlich bedarf diese Argumentation eines unbegrenzten Wirtschaftswachstums und einer Sicherstellung eines stabilen Wertentwicklungsverhältnisses. Dieses Prinzip läuft Gefahr, umstrittene Ungerechtigkeitsmechanismen zu legitimieren, da den jeweils Schlechtestgestellten per se der Vorrang gegeben würde, selbst wenn ein gewisser Grad an Akzeptabilität der Lebensumstände erreicht wäre. Die Durchsetzbarkeit und Praktikabilität stehen infrage (vgl. 
Gesang 2011, 55ff.). Neben diesen drei Prinzipien gibt es zahlreiche weitere Diskurspunkte, die das Feld der Klimaethik und die potenziellen Moralisierungstendenzen facettenreicher werden lassen. Die Moral ist hier nicht mehr nur als eindimensional oder dichotom zu verstehen.

\subsection{Erste Zusammenführungen und weiterführende Betrachtungen}

An diesem Punkt konnte bereits gezeigt werden, dass (a) der Klimawandel moralischen Fragen und Bewertungen unterliegt (vgl. Roser und Seidel 2015, 15-24), (b) der Klimawandel durch die Vernetzung von grundlegenden Gerechtigkeitsfragen, moralischen Ansprüchen und zum Teil divergierenden sowie fusionierenden Problematiken in den räumlichen und zeitlichen Dimensionen hinsichtlich der Ursache-WirkungsRelationen ein komplexes moralisches Gefüge aufwirft, das (c) zu vielfältigen Moralisierungstendenzen führen kann, und dass (d) der moralische Status des Klimawandels und die daraus folgenden Ableitungen für das Handeln des Menschen und der Gesellschaft weitere Fragen aufwerfen, die einer Prüfung und Reflexion bedürfen. Die Moralisierungstendenzen gehen demnach mit einer Form der reflexiv-kritischen und prüfenden ethischen Haltung einher, wodurch die Moral als »die Gesamtheit der akzeptierten und durch Tradierung stabilisierten Verhaltensnormen einer Gesellschaft « (Jüssen 2019, 149) einen differenzierteren Fokus erfährt. Die Ethik ist dabei die prüfende Instanz, die »nach der rationalen Begründung moralischer Urteile und Prinzipien sucht « (Vašek 2019, 17). Neben den ansatzweise explizierten Fragen der Gerechtigkeit (vgl. dazu auch Roser 2016; Hohl 2016; Ekardt 2012) beschäftigt sich der klimaethische Diskurs mit einem basalen Momentum der Moral: Verantwortung.

»Moralische Verantwortung schreiben wir einer Person in der Regel dann zu, wenn sie auch anders hätte handeln können, also zum Beispiel die schädliche Handlung hätte unterlassen können. Dies bedingt, dass die Person zudem über Wissen verfügt, beispielsweise dass sie mit ihrer Handlung etwas Schlechtes tut « (von Allmen 2018).

Das Problem der Verantwortung angesichts des Klimawandels subsumiert, transformiert, integriert und katalysiert die einzelnen Bestimmungsstücke innerhalb des klimaethischen Diskurses. An dem Begriff und Prinzip Verantwortung kann die Entfaltung der spezifisch das klimaethische Selbst konstruierenden Dissonanzen, Diffusionen, Anforderungen, Verflechtungen und Schwierigkeiten der Moralisierung des Selbst skizziert werden. Dazu sollen zwei exemplarische Konnotationsdomänen des Verantwortungsbegriffs und die dazugehörigen moralisierenden Tendenzen, die das Selbst idealtypisch zu konstruieren erlauben, aufgezeigt werden. Die individuelle Verantwortung und die 
Langzeitverantwortung scheinen zwei passende Konzepte zu sein, um die klimaethische Fassung des Selbst konstruieren zu können.

\section{Das klimaethische Selbst - Eine verantwortungsbasierte Subjektivierungsform im moralischen Klimawandeldiskurs}

Insgesamt stellt die geforderte, moralisierende Tendenz der Ausweitung des Geltungsbereichs der Moral und ethischer Diskurse auf globale Phänomene und kommende Generationen sowie die grundsätzliche Fokusverschiebung weg von sich selbst hin zu geografisch und zeitlich weit entfernten Individuen das Selbst vor große Herausforderungen (vgl. Straub und Ruppel 2017, 8f.). Diese zeigen sich insbesondere in der dargestellten klimaethisch-gerechtigkeitstheoretischen und grundlegend moralischen Debatte (vgl. Roser und Seidel 2015, 23ff.). Vereint werden sie in dem Wirkmechanismus der Verantwortung, die als theoretisch-klärender und konstituierender Katalysator zur Konstruktion des klimaethischen Selbst fungiert. Dazu soll sich in einem ersten Schritt dem Konzept der individuellen Verantwortung genähert werden, um dann den Bogen zur Langzeitverantwortung zu spannen. Die exemplarische Fokussierung auf diese beiden Konnotationsdomänen ist zwangsläufig eine Form der Reduktion, soll aber aufzeigen, dass neben der Vielzahl an gestellten verantwortungsbasierten Ansprüchen an das Individuum diese durch Vernetzungen verbunden sind. Zudem dient dies der idealtypischen Konstruktion des klimaethischen Selbst, welche an eine die soziale Wirklichkeit ideal-modellhaft erfassende Konstruktion eines Idealtypus nach Weber (1951) angelehnt ist. Eine Schnittmenge im Verständnis der Konstruktion eines Idealtypus liegt insofern vor, als dass die Konstruktion des klimaethischen Selbst von der Wirklichkeit abstrahiert und versucht, die alltäglichen Moralisierungstendenzen als Formen sozialer Ansprüche zu sehen, die in Rekurs auf klimaethische, gerechtigkeitstheoretische und verantwortungstheoretische Diskurse zu einem tendenziell utopischen, ethischen Ideal in Form des klimaethischen Selbst theoretisierend idealisiert werden (vgl. Dieckmann 1967; Merz-Benz und Wagner 2007). Dabei ist dieses Ideal eines klimaethischen Selbst »keineswegs allgemeingültig« (Dieckmann 1967, 29).

\subsection{Zur individuellen Verantwortung}

Die grundlegende Frage klimaethischer Verantwortungsdiskurse lässt sich wie folgt formulieren: »Wie weit muss sich das Individuum verpflichtet fühlen, zur Emissionsminderung beizutragen, etwa durch geeignete Änderungen in Lebensstil und Konsumverhalten, eventuell auch durch aktive Beteiligung an und Einwirkung auf politische 
Entscheidungsprozesse? «(Birnbacher 2016, 128) Die Begründung einer individuellen Verantwortung ist dabei ein schwieriges Unterfangen (vgl. von Allmen 2018; Roser und Seidel 2015). Grundsätzlich kann in dieser Debatte das »contributor's dilemma « (Birnbacher 2016, 129) herangezogen werden. Der individuelle Beitrag einer Person zur Erreichung eines kollektiven Ziels erscheint in der Gesamtbetrachtung als so gering, dass der potenzielle und faktische Gewinn des individuellen Tuns mit den für diese Person entstehenden Aufwänden in keinem Verhältnis steht. »Bei dem Subjekt der Klimaverantwortung handelt es sich mit aller Wahrscheinlichkeit nicht um ein einzelnes Individuum, denn der Klimawandel ist ein synergetischer Verantwortungsgegenstand « (Sombetzki 2014, 232). Wallimann-Helmer (2017, 215) spricht sich ebenfalls für eine Fokussierung auf kollektive, staatliche Verantwortungsgefüge aus: $\gg[\mathrm{A}]$ uf der einen Seite sind Individuen und zum Teil auch Unternehmen tendenziell epistemisch überfordert, wenn es darum geht, das eigene Handeln in klimaneutralen Bahnen zu lenken.« Mit dieser »Schieflage « muss das Individuum tagtäglich in praktischen Alltagshandlungen umgehen. Dabei ist aber $\gg[$ d] ie Frage nach Sinn, Berechtigung und Reichweite individueller Verantwortung für kollektive Schädigungen « (Birnbacher 2016, 129) eine wesentlich differenzierte. Birnbacher (2016) erörtert die Frage, inwieweit individuelles Verhalten einen Einfluss auf moralisch problematisches Kollektivhandeln hat, entlang konsequenzialistischer Überlegungen. In dieser Argumentationslogik zählen primär die abzusehenden Folgen für die moralische Wertung und Reichweite moralischer Folgenverantwortung eines Individuums. Er argumentiert, dass es für ein Vorhandensein individueller Verantwortung ausreichend ist, dass mit einer gewissen Wahrscheinlichkeit beziehungsweise einer potenziellen Möglichkeit ein klimaschädlicher Effekt verstärkt wird. Somit hängen der tatsächliche Effekt und das tatsächliche Verhalten in keiner stringenten Kausalität mit dem Problem an sich zusammen, aber allein die Möglichkeit, dass potenzielles Verhalten zu potenzieller Schädigung der Klimaverhältnisse oder aber zu positiver Beeinflussung des Klimawandels führen kann, rechtfertigt die moralische Verantwortung des Individuums. Dies bedeutet weiterhin, dass Qualität und Quantität individueller Verantwortung im Sinne eines Ausmaßes des Wirkspektrums und der konkreten Übernahme der Verantwortung des Einzelnen dennoch kontextualisiert an gewisse eigene Möglichkeiten gebunden sind (vgl. Birnbacher 2016, 130ff.). Birnbacher $(2016,132)$ umschreibt dies als eine Art von Interdependenz, denn »[d]ie Reichweite der Verantwortung [eines Individuums] bemisst sich nach seiner Wirkmächtigkeit.« Dies ist ein verbindender Punkt zur Idee des klimaethischen Selbst, denn mit zunehmender Autonomie und zunehmendem Bewusstsein wächst individuelle Verantwortung. In einem maßgeblich an Rationalität orientierten Zugang zu Verantwortung, welcher das idealisierte klimaethische Selbst durchaus kennzeichnet, erscheint neben der Bedingung, dass das Subjekt verschiedenen Bereichen und Effekten im Klimawandeldiskurs begegnen kann, unter anderem auch 
ein mehr oder weniger explizites Wissen um Handlungsfolgen relevant zu sein. »Jeder Zuwachs an Folgenkenntnis beziehungsweise an Wissen über Chancen und Risiken trägt dazu bei, bei bestehenden Einflussmöglichkeiten die Handlungsmacht und damit die Verantwortung zu erweitern « (Birnbacher 2016, 132). So ergeben sich verschiedene Qualitäten an Verantwortlichkeit und quantitative Wirkspielräume, die je nach situativer (Lebens-)Lage ein individuelles mitverantwortliches, eigenaktives Handeln ermöglichen und konstituieren. Birnbacher (2016, 138-149) stellt dabei den potenziellen Erwartungswert der Folgen als grundlegend für die ethische Bewertung und Begründung der Übernahme individueller Verantwortung heraus. Diese Argumentation ist nicht unumstritten (vgl. Hohl 2017, Kap. 4), zeigt aber, dass sich in ersten Schritten eine individuelle Verantwortung des Einzelnen begründen lässt. Hohl (2017, 152) kommt im Diskurs mit dieser Sichtweise zu dem Schluss, dass diese einen Ansatz für weitere Begründungslinien der individuellen Verantwortung angesichts eines kollektiv verursachten Übels darstellen kann. Zu einer ähnlichen Ansicht gelangen auch Braun und Baatz (2017), die ein umfassendes Konzept der Klimaverantwortung vorlegen, welches die Relationalität zwischen dem Menschen als aktives und reflexives, in der Natur verortetes handelndes »Verantwortungssubjekt « und dem Klimawandel als »Verantwortungsobjekt « betont. Sie betrachten die individuelle Verantwortung des Menschen in Kombination mit einer zunehmend gesellschaftlichen Verantwortung im sozialen Kontext (vgl. Braun und Baatz 2017, 866). Diese Kopplung lässt den Transfer zur Idee der Langzeitverantwortung zu.

\subsection{Zur Langzeitverantwortung}

Grundsätzlich korrespondiert sie mit der Überlegung der (Zukunfts-)Verantwortung gegenüber folgenden und »fernen Generationen « (Gethmann 2008, 10). Sturma (2008, 51) bringt den Begriff auf eine fassbare Formel: »Verantwortung beruht auf einer normativen Zuschreibung. Langzeitverantwortung äußert sich dementsprechend in einem asymmetrischen normativen Verhältnis zwischen lebenden Personen als Subjekten von Zuschreibungen und zukünftigen Personen als Adressaten der Zuschreibungen.«In dieser Sicht der Verantwortlichkeit kann ein individueller Beitrag zur Langzeitverantwortung ausgemacht werden, der zusätzliche Anforderungen an das Selbst stellt, aber auch klärende Perspektiven eröffnet. Der Fokus der klimaethischen Diskussion liegt auf einer zukunftsorientierten Verantwortung (vgl. Roser und Seidel 2015, Kap. 3) und integriert die Problematik der individuellen Pflichten sowie Verantwortungsqualitäten und -quantitäten. Gethmann (2008, 17ff.) argumentiert, dass die Individuen der jetzigen Generation durchaus in den Verantwortungsdiskurs einbezogen werden müssen, da sie gegenüber künftigen Personen gewisse Verpflichtungen in ihren jeweiligen potenziell 
gestaltbaren Lebensentwürfen und Situationen haben, die wiederrum in Bezug zu den potenziellen Bedürfnissen und Ansprüchen der folgenden Generationen zu setzen sind. Entscheidend bei dieser Konzeption ist, dass auch zukünftige Menschen ein $\gg$ Interesse an Mindeststandards für gesundes Leben, körperliche Integrität und an Menschenrechten « (Sturma 2008, 52) haben. Diese Grundlagen menschlicher Lebensformen sind damit erhaltungswürdig und ebnen den Weg für die Übernahme einer Langzeitverantwortung des Einzelnen. Sturma $(2008,57)$ spricht einen weiteren, zum klimaethischen Selbst hinführenden Punkt an: Die Reflexion gegenwärtiger Personen ist ein grundlegender Faktor in der Übernahme einer Langzeitverantwortung, denn

»Personen haben aus ihrem aufgeklärten Selbstverständnis heraus gute Gründe, sich nicht zumuten zu wollen, Raubbau oder Rücksichtslosigkeit gegenüber nachfolgenden Personen auszuüben, auch wenn diese sie faktisch nie zur Rechenschaft ziehen können. Die Verantwortung gegenüber künftigen Personen wäre insofern eine Verantwortung sich selbst gegenüber.«

Diese selbstreferenzielle Überlegung (vgl. Musholt 2016) bereitet einen argumentativen Brückenschlag zum klimaethischen Selbst.

\subsection{Das klimaethische Selbst}

Das klimaethische Selbst als idealtypisches Konstrukt ist gekennzeichnet von jener reflexiven Selbstbezüglichkeit und wird als » absichtlich-aktive[r] Gestalte[r] der Umwelt, [seiner] Person und damit [als] intentionale[r] Gestalter [seiner] Entwicklung « (Greve 2007, 322) aufgefasst. Damit wird das klimaethische Selbst als ein reflexives Momentum entworfen, das geprägt ist von Integration und Revision (vgl. Greve und Thomsen 2019, 165). Diese Fähigkeit zur (Selbst-)Reflexivität und kontextgebundenen Adaption erlaubt die Integration einer ethischen Praxis und die stete Prüfung moralischer Ansprüche und ihrer Umsetzungsmöglichkeiten. Das klimaethische Selbst als eine idealtypische Facette des Selbst ist diskursiv-reflexiv verfasst. Es ist durch die Flexibilität und »merkliche intrapersonale Variabilität « (Greve und Thomsen 2019, 165) charakterisiert, da es an sich wandelnde klimaethische und verantwortungstheoretische Diskurse, die als Prüfpunkte normativer Moralisierungstendenzen fungieren, rückgebunden ist (Punkt 2 und 3). Damit ist es zudem synergetisch und kritisch-reflexiv, da es die moralisierenden Tendenzen des Alltags, des öffentlichen Lebens und anderer Bereiche, die mitunter in absoluter Weise auf ein klimafreundliches Ideal referieren und das Individuum zu einem nachhaltigen und klimaneutralen Handeln befähigen sollen (vgl. Straub und Ruppel 2017), erfasst, diskutiert, prüft und in adaptiver Wei- 
se integriert oder zurückweist sowie modifiziert. Das klimaethische Selbst ist somit geprägt von der Grundidee der Ethik: dem reflexiven Prüfen von moralischen Ansprüchen, um situativ und kontextgebunden unter Einbezug verschiedener Bewertungs- und Entscheidungsmodi diese Moralisierungstendenzen zu betrachten. Das klimaethische Selbst ist der Reflexionskern, der ein dynamisches und gleichzeitig (mehr oder weniger) stabiles Selbst angesichts der Herausforderungen der Klimawandelproblematik und all ihrer einhergehenden Anforderungen stützen kann (vgl. Greve 2007, 327).

Durch diese Betrachtung wurden folgende Bedeutungsdomänen herausgearbeitet: (a) das Bestimmungsstück, dass sich das Subjekt reflexiv mit Moralisierungstendenzen auseinandersetzen kann und (b) dass das klimaethische Selbst dieser sich selbst thematisierende Reflexionskern sein kann und sollte, um sich der Mitwelt und sich selbst gegenüber diskursiv und prüfend verhalten zu können. Das klimaethische Selbst kann (c) idealiter als ein von Offenheit geprägtes verstanden werden, »[das sich] möglichst unvoreingenommen die Bandbreite der Möglichkeiten [...] erarbeitet und daraus mögliche Selbstentwürfe formuliert « (Bayer und Gollwitzer 2000, 212). Die Offenheit besitzt das klimaethische Selbst (d), da es, aus verantwortungs- und gerechtigkeitstheoretischen sowie ethischen Überlegungen gespeist, sich im Diskurs mit seiner Mitwelt und durch Reflexion mit moralisierenden Aspekten auseinandersetzen kann. Das Abwägen (e) klimabezogener Moralansprüche durch klimaethische Reflexion und Kritik machen diese relativierbarer. Das klimaethische Selbst (f) akzentuiert und integriert das Soll-Selbst, das ideale Selbst und das mögliche Selbst (vgl. Morf und Koole 2014, 156). Es umfasst (g) eine aktionale und » wertbildende « Komponente, welche die eigene Entwicklung und den (reflexiven) Umgang mit Moralisierungstendenzen gestaltbar werden lässt (vgl. Greve 2007, 329). Weiterhin ist es eine adaptive und kritisch-konstruktive Diskurspraxis (h), die vom Subjekt reflexiv und prüfend angeeignet und vollzogen werden kann. Die Auswirkungen dieser Formung des Selbst auf den Klimawandel können an dieser Stelle nicht eruiert werden. Die klimaethische Selbstthematisierung entlang moralischer und ethischer Ansprüche sowie abgewogener Normmaßstäbe stellt einen potenziellen Reflexionsprozess dar, der einen orientierenden Beitrag für das Individuum im anthropogenen Verantwortungskomplex des Klimawandels leisten kann.

\section{Abschließende Betrachtungen und ausblickhafte Überlegungen}

Resümierend kann festgehalten werden, dass die Moral eine Form der Stabilität und gewissen idealen Geltungshoheit markiert, die Ethik wiederum die notwendige, prüfende, revidierende und kritische Reflexion anzeigt und ermöglicht (vgl. Berger 2011). »Während ihre frühere Funktion darin lag, rationale Rechtfertigungen für Moral zur Verfügung zu stellen, sollte Ethik zukünftig vor übertriebener Moralisierung warnen « 
(Besio und Pronzini 2018, 81). Das ideale, klimaethische Selbst ist in diesem Kontext eine Facette, die den Moralisierungstendenzen in mehrfacher Hinsicht in einer diskursiven Praxis begegnet und kontextuell sowie inhaltlich mit diesen verbunden ist. (a) Die moralischen Anforderungen werden im Klimawandeldiskurs an das Selbst herangetragen und im Sinne von normativen Soll-Sätzen gefordert. Diese Ansprüche können zu einer Überlastung und Überforderung bis hin zur Abneigung und Ablehnung führen, aber ebenso ist die Integration dieser Diskurse in den Selbstbildungsprozess möglich. (b) Die klimaethische Betrachtungsweise und argumentativ-kritische Prüfung der moralischen Tendenzen erlauben durch die Rückführung der Moralansprüche auf die ihnen zugrunde liegenden Theorien und Annahmen einen konstruktiven und klärenden Umgang mit diesen. Ein so verstandenes proaktives und rationalistisches, ideales klimaethisches Selbst (c) ermöglicht in Bezug auf den Umgang mit klimamoralischen Forderungen in Form einer ethischen Reflexion und Diskussion die Einordnung, Anpassung, Revision, Begründung und Positionierung sowie Gestaltung des Handelns (vgl. Berger 2011). Die Stilisierung des klimaethischen Selbst (d) ist ferner eine Form der Idealisierung ethisch-rationaler Existenzmöglichkeiten, die als wünschenswerte, idealtypische Facette des Selbst abstrahiert werden und die es in ihren normativen Zwängen und Ansprüchen perspektivisch zu reflektieren gilt. Das klimaethische Selbst soll (e) als ein dynamisches Momentum der persönlichen Entwicklung gesehen werden (vgl. Morf und Koole 2014, 153), in dem theoretische und reflexive sowie selbstreferenzielle Diskursmomente in Bezug zum Klimawandel zusammenlaufen, da philosophische und klimaethische Betrachtungsweisen dies durch den diskursiven und prüfenden Erwägungs- und Abwägungscharakter ermöglichen können, denn

»[d]ass Werte und Normen kontingent sind, bedeutet keineswegs, dass wir nicht Vernunftgründe geltend machen können, um dafür zu argumentieren, sie anzuerkennen und gelten zu lassen, für allgemein verbindliche zu halten und ihnen in unserem Handeln gerecht zu werden. [...] Wir müssen Werte immer wieder neu auslegen, um sie in konkreten Situationen als Leitfaden und Richtschnur unseres Handelns und Lebens konkretisieren und etablieren zu können « (Straub und Ruppel 2017, 17).

Das hier konstruierte Ideal eines klimaethischen Selbst vermag dies als sich selbstthematisierende, reflexiv-diskursive Subjektivierungsform theoretisch zu leisten. Die nachhaltigkeitsorientierte, empirische Bewährung dieser idealtypischen Konstruktion bleibt indes fraglich. Aus dieser idealtypischen Konstruktion ergeben sich deswegen mannigfaltige Fragen für die empirische und moralpsychologische Forschung sowie für die Forschungsschwerpunkte der Psychologie des Selbst, deren Bearbeitung an weitere transdisziplinäre Forschungsfelder anschließt. Wie erleben Personen die klimamoralischen Ansprüche und wie integrieren sie diese in ihr Selbstkonzept? Wie wirken 
dynamische Entwicklungstendenzen innerhalb des Selbstbildungsprozesses angesichts der klimaethischen Reflexion? Wie differenzieren Personen in ihren Selbstthematisierungen mögliche, erwünschte und ideale Selbstentwürfe hinsichtlich des Klimawandels aus? Mit der idealtypischen Konstruktion des klimaethischen Selbst im theoretisch-normativen und verantwortungsorientierten Diskurs soll zur Beantwortung dieser und weiterer Fragen eingeladen werden.

\section{Literatur}

Althusser, Louis. 2010. Ideologie und ideologische Staatsapparate. 1. Halbband: Michel Verrets Artikel über den »studentischen Mai«. Ideologie und ideologische Staatsapparate. Notiz über die ISAs. Hamburg: VSA.

Bayer, Ute C. und Peter M. Gollwitzer. 2000. »Selbst und Zielstreben«. In Psychologie des Selbst, hrsg. v. Werner Greve, 208-25. Weinheim: Beltz, Psychologie-Verlag-Union.

Bendig, Rebekka. 2018. Handlungskompetenzen entwickeln am Lerngegenstand Kinderrechte. Globales Lernen in Kooperation von Schule, Zivilgesellschaft und Jugendarbeit. Wiesbaden: Springer VS.

Berger, Hartwig. 2011. »Ethik im Klimawandel«. Zugriff 06.05.2020. https://www.hartwig-berger. de/cms/ethik-im-klimawandel/.

Besio, Cristina und Andrea Pronzini. 2014. »Morality, Ethics, and Values Outside and Inside Organizations: An Example of the Discourse on Climate Change«. Journal of Business Ethics 119 (3): 287-300.

Besio, Cristina und Andrea Pronzini. 2018. »Moral, Ethik und Werte außerhalb und innerhalb von Organisationen. Das Beispiel des Diskurses über Klimawandel«. In Moral und Innovation in Organisationen, hrsg. v. Cristina Besio, 59-81. Wiesbaden: Springer Fachmedien.

Beyerl, Katharina. 2010. »Der Klimawandel in der psychologischen Forschung «. In Der Klimawandel. Sozialwissenschaftliche Perspektiven, hrsg. v. Martin Voss, 247-65. Wiesbaden: VS Verlag für Sozialwissenschaften.

Birnbacher, Dieter. 2016. Klimaethik. Nach uns die Sintflut? Stuttgart: Reclam.

Bischof, Norbert. 2012. Moral. Ihre Natur, ihre Dynamik und ihr Schatten. Wien, Köln \& Weimar: Böhlau Verlag.

Boehme-Neßler, Volker. 2019. »Moralsteuern für das Klima?« ZEIT Online, 15. August. Zugriff 04.05.2020. https://www.zeit.de/politik/deutschland/2019-08/politik-moral-trennung-klima politik-fleischsteuer.

Böhm, Gisela. 2008. »Wahrnehmung und Bewertung von Umweltrisiken«. In Enzyklopädie der Psychologie: Umweltpsychologie - Band 1. Grundlagen, Paradigmen und Methoden der Umweltpsychologie, hrsg. v. Ernst-Dieter Lantermann und Volker Linneweber, 501-32. Göttingen: Hogrefe.

Braun, Florian und Christian Baatz. 2017. „Klimaverantwortung«. In Handbuch Verantwortung, hrsg. v. Ludger Heidbrink, Claus Langbehn, und Janina Loh, 855-86. Wiesbaden: Springer VS.

Bröckling, Ulrich. 2007. Das unternehmerische Selbst. Soziologie einer Subjektivierungsform. Frankfurt a. M.: Suhrkamp.

Bruder, Klaus-Jürgen. 2005. „Selbstthematisierung«. Journal für Psychologie 13 (3): 192-211.

Bundesministerium für Bildung und Forschung (BMBF). 2019. Bildung und Forschung als Schlüssel für eine nachhaltige Zukunft - Ressortbericht zur nachhaltigen Entwicklung. Bonn: BMBF. 
Burkart, Günter. 2006. »Einleitung«. In Die Ausweitung der Bekenntniskultur - neue Formen der Selbstthematisierung?, hrsg. v. Günter Burkart, 7-40. Wiesbaden: VS Verlag für Sozialwissenschaften.

Compagna, Diego. 2020. »Das `Atlas-Subjekt und neue Formen von Subjektivierung im Zeitalter der Nachhaltigkeit«. In Nach haltig Leben und Wirtschaften. Management Sozialer Innovationen als Gestaltung gesellschaftlicher Transformation, hrsg. v. Hans-Werner Franz, Gerald Beck, Diego Compagna, Peter Dürr, Wolfgang Gehra und Martina Wegner, 33-52. Wiesbaden: Springer Fachmedien.

Dieckmann, Johann. 1967. »Die Rationalität des Weberschen Idealtypus«. Soziale Welt 18 (1): 29-40.

Diefenbacher, Hans. 2012. Gerechtigkeit und Nachhaltigkeit. Zum Verhältnis von Ethik und Ökonomie. Darmstadt: WBG.

Ekardt, Felix. 2012. »Klimagerechtigkeit: Neue universalistische Diskursethik, neue Freiheitstheorie, neue Abwägungstheorie. Eine ethische und menschenrechtliche Analyse - und eine Alternative zur Kosten-Nutzen-Analyse«. In Klimagerechtigkeit. Ethische, rechtliche, ökonomische und transdisziplinäre Zugänge, hrsg. v. Felix Ekardt, 157-204. Marburg: Metropolis.

Foucault, Michel. 1993. »About the Beginning of the Hermeneutics of the Self: Two Lectures at Dartmouth«. Political Theory 21 (2): 198-227.

Geimer, Alexander und Steffen Amling. 2019. »Subjektivierungsforschung als rekonstruktive Sozialforschung vor dem Hintergrund der Governmentality und Cultural Studies. Eine Typologie der Relation zwischen Subjektnormen und Habitus als Verhältnisse der Spannung, Passung und Aneignung". In Subjekt und Subjektivierung. Empirische und theoretische Perspektiven auf Subjektivierungsprozesse, hrsg. v. Alexander Geimer, Steffen Amling und Saša Bosančić, 19-42. Wiesbaden: Springer Fachmedien.

Geimer, Alexander, Steffen Amling und Saša Bosančić. 2019. „Einleitung: Anliegen und Konturen der Subjektivierungsforschung". In Subjekt und Subjektivierung. Empirische und theoretische Perspektiven auf Subjektivierungsprozesse, hrsg. v. Alexander Geimer, Steffen Amling und Saša Bosančić, 1-15. Wiesbaden: Springer Fachmedien.

Gesang, Bernward. 2011. Klimaethik. Berlin: suhrkamp taschenbuch wissenschaft verlag.

Gethmann, Carl Friedrich. 2008. »Wer ist der Adressat der Langzeitverpflichtung?« In Langzeitverantwortung. Ethik. Technik. Ökologie, hrsg. v. Carl Friedrich Gethmann und Jürgen Mittelstrass, 10-22. Darmstadt: WBG.

Greve, Werner. 2007. "Selbst und Identität im Lebenslauf«. In Entwicklungspsychologie der Lebensspanne. Ein Lehrbuch, hrsg. v. Jochen Brandstädter und Ulman Lindenberger, 305-36. Stuttgart: Kohlhammer.

Greve, Werner und Maren Etzold. 2018. „Die Psychologien des Selbst - Konvergente Perspektiven auf ein vielfältiges Konzept«. In Das Selbst. Psychologische Perspektiven, hrsg. v. Werner Greve, 7-21. Hildesheim: Universitätsverlag Hildesheim.

Greve, Werner und Tamara Thomsen. 2019. Entwicklungspsychologie. Eine Einführung in die Erklärung menschlicher Entwicklung. Wiesbaden: Springer.

Hohl, Sabine. 2016. »Zukünftige Generationen«. In Handbuch Gerechtigkeit, hrsg. v. Anna Goppel, Corinna Mieth und Christian Neuhäuser, 478-83. Stuttgart: J. B. Metzler Verlag.

Hohl, Sabine. 2017. Individuelle Verantwortung für kollektive verursachte Übel. Münster: mentis Verlag.

Jüssen, Gabriel. 2019. »Moral, moralisch, Moralphilosophie«. In Historisches Wörterbuch der Philosophie. Band 6: Mo-O, hrsg. v. Joachim Ritter und Karlfried Gründer, 149-51. Darmstadt: WBG.

Keller, Rainer. 2008. Wissenssoziologische Diskursanalyse. Grundlegung eines Forschungsprogramms. 2. Aufl. Wiesbaden: VS Verlag für Sozialwissenschaften.

Kessler, Thomas und Immo Fritsche. 2018. Sozialpsychologie. Wiesbaden: Springer Fachmedien. 
Knobloch, Clemens. 2015. »Moralisierung in der öffentlichen Kommunikation«. Zeitschrift für Literaturwissenschaft und Linguistik 45 (177): 167-84.

Liessmann, Konrad Paul. 2019. „Das Klima markiert die neue Gretchenfrage der Moral«. Neue Züricher Zeitung [Kolumne], 10. September. Zugriff 11.05.2020. https://www.nzz.ch/meinung/ kolumnen/das-klima-markiert-die-neue-gretchenfrage-der-moral-ld.1507395.

Lörcher, Ines. 2019. »Wie kommt der Klimawandel in die Köpfe? Ein Forschungsüberblick zur Perzeption des Klimawandels und der Nutzung, Aneignung und Wirkung von medialer und interpersonaler Kommunikation sowie direkten Erlebnissen«. In Klimawandel im Kopf. Studien zur Wirkung, Aneignung und Online-Kommunikation, hrsg. v. Irene Neverla Monika Taddicken, Ines Lörcher und Imke Hoppe, 53-76. Wiesbaden: Springer Fachmedien.

Luhmann, Niklas. 1993. Gesellschaftsstruktur und Semantik. Studien zur Wissenssoziologie der modernen Gesellschaft. Bd. 3. Frankfurt a. M.: Suhrkamp.

Merz-Benz, Peter-Ulrich und Gerhard Wagner. 2007. »Idealtypus und Verstehen. Max Webers Logik der Handlungsdeutung «. In Jahrbuch für Soziologiegeschichte, hrsg. v. Carsten Klingemann, 53-66. Wiesbaden: VS Verlag für Sozialwissenschaften.

Morf, Carolyn C. und Sander L. Koole. 2014. »Das Selbst«. In Sozialpsychologie, 6., vollst. überarb. Aufl., hrsg. v. Klaus Jonas, Wolfgang Stroebe und Miles Hewstone, 141-96. Berlin \& Heidelberg: Springer-Verlag (Springer-Lehrbuch).

Musholt, Kristina. 2016. „Durch mich selbst zur Identität?« Blogbeitrag, philosophie.ch. Swiss Portal for Philosophy, 27.11.2016. Zugriff 22.05.2020. https://www.philosophie.ch/philosophie/ highlights/mensch/durch-mich-selbst-zur-identitaet.

Neuhäuser, Christian. 2020. »Kluge Moral ist nicht moralistisch«. Deutschlandfunk Kultur, Sein und Streit, 02.02.2020. Zugriff 08.05.2020. https://www.deutschlandfunkkultur.de/philosoph-chr istian-neuhaeuser-zur-klimadebatte-kluge-moral.2162.de.html?dram:article_id=469269.

Pfister, Hans-Rüdiger, Helmut Jungermann und Katrin Fischer. 2017. Die Psychologie der Entscheidung. Eine Einführung. 4. Aufl. Berlin \& Heidelberg: Springer-Verlag.

Rahmstorf, Stefan und Hans Joachim Schellnhuber. 2019. Der Klimawandel. Diagnose, Prognose, Therapie. 9. Aufl. München: C.H. Beck.

Rehmann-Sutter, Christoph. 2019. „Klimawandel - und die Philosophie?« Beitrag, philosophie.ch. Swiss Portal for Philosophy, Serie zur Ethik des Klimawandels, 16.07.2019. Zugriff 22.05.2020. https://www.philosophie.ch/artikel/2018/klimawandel-und-die-philosophie.

Roser, Dominic. 2016. »Klima und Umwelt«. In Handbuch Gerechtigkeit, hrsg. v. Anna Goppel, Corinna Mieth und Christian Neuhäuser, 406-13. Stuttgart: J. B. Metzler Verlag.

Roser, Dominic und Christian Seidel. 2015. Ethik des Klimawandels. Eine Einführung. 2. Aufl. Darmstadt: WBG.

Schaper, Ulrich. 2015. Klimawandel und Lebenswirklichkeit. Münster: MV Wissenschaft.

Schmidt, Andreas. 2015. »Moralvorstellungen in der öffentlichen Debatte: Konzeptionelle und methodische Überlegungen zu Relevanz und empirischer Untersuchung «. SCM 4 (2): 69-134.

Schmidt, Udo. 2019. »Die dunkle Seite der Klima-Moral«. Podcast, 23.09.2019, 18:25 Uhr, 00:03:02, NDR Info, Auf ein Wort. Zugriff 08.05.2020. https://www.ndr.de/nachrichten/info/Glosse-Die -dunkle-Seite-der-Klima-Moral,audio564706.html.

Schönwiese, Christian-D. 2019. Klimawandel kompakt. Ein globales Problem wissenschaftlich erklärt. 2. durchges. U. korr. Aufl. Stuttgart: Borntraeger.

Schroer, Markus. 2006. „Selbstthematisierung. Von der (Er-)Findung des Selbst und der Suche nach Aufmerksamkeit«. In Die Ausweitung der Bekenntniskultur - neue Formen der Selbstthematisierung?, hrsg. v. Günter Burkart, 41-72. Wiesbaden: VS Verlag für Sozialwissenschaften.

Sombetzki, Janina. 2014. Verantwortung als Begriff, Fähigkeit, Aufgabe. Eine Drei-Ebenen-Analyse. Wiesbaden: Springer Fachmedien. 
Sterman, John D. 2008. »Risk Communication on Climate: Mental Models and Mass Balance«. Science 322 (5901): 532-33.

Straub, Jürgen und Paul Sebastian Ruppel. 2017. »Vegan und kerosinfrei. Eine moralische Subjektivierungsform im 21. Jahrhundert. Einführung in den Themenschwerpunkt«. psychosozial 40 (2), Nr. 148: 5-31.

Sturma, Dieter. 2008. „Die Gegenwart der Langzeitverantwortung«. In Langzeitverantwortung. Ethik. Technik. Ökologie, hrsg. v. Carl Friedrich Gethmann und Jürgen Mittelstrass, 40-57. Darmstadt: WBG.

Vašek, Thomas. 2019. „Eine besondere Art des Miteinander«. HOHE Luft, Ausgabe 2/2019: 14-21.

Von Allmen, Kathrin. 2018. „Bin ich moralisch verantwortlich für den Klimawandel? Klimaethik und Verantwortung«. Blogbeitrag, philosophie.ch. Swiss Portal for Philosophy, Philosophie aktuell, 05.02.2018. Zugriff 11.05.2020. https://www.philosophie.ch/philosophie/highlights/ philosophie-aktuell/bin-ich-moralisch-verantwortlich-fuer-den-klimawandel.

Wallimann-Helmer, Ivo. 2017. »Kollektive Verantwortung für den Klimaschutz«. Zeitschrift für praktische Philosophie 4 (1): 211-38.

Weber, Max. 1951. Gesammelte Aufsätze zur Wissenschaftslehre. 2. Aufl. Tübingen: J.V. B. Mohr (Paul Siebeck).

Willems, Herbert und Sebastian Pranz. 2008. „Formationen und Transformationen der Selbstthematisierung. Von der unmittelbaren Interaktion zum Internet«. In Weltweite Welten. InternetFigurationen aus wissenssoziologischer Perspektive, hrsg. v. Herbert Willems, 189-222. Wiesbaden: VS Verlag für Sozialwissenschaften.

\section{Die Autorin}

Alexandria Krug, 2018 Erstes Staatsexamen Lehramt an Grundschulen an der Universität Leipzig, seit 2019 wissenschaftliche Mitarbeiterin und seit 2020 Doktorandin an der Universität Leipzig. Forschungsbereiche ihrer Dissertation: Klimaethik, Verantwortung, Klimagerechtigkeit, mentale Modelle zum Klimawandel von Grundschulkindern, Schulgartenarbeit und bildungstheoretische Diskurse sowie das Philosophieren mit Kindern.

Kontakt: Alexandria Krug, Universität Leipzig, Erziehungswissenschaftliche Fakultät, Institut für Pädagogik und Didaktik im Elementar- und Primarbereich, Grundschuldidaktik Sachunterricht unter besonderer Berücksichtigung von Naturwissenschaft und Technik, Marschnerstraße 31, 04109 Leipzig; E-Mail: alexandria.krug@uni-leipzig.de 